DIGITALCOMMONS

— @WAYNESTATE -

Journal of Modern Applied Statistical Methods

Volume 10 | Issue 2

Article 26

$11-1-2011$

\title{
Height-Diameter Relationship in Tree Modeling Using Simultaneous Equation Techniques in Correlated Normal Deviates
}

\author{
S. O. Oyamakin
}

Forestry Research Institute of Nigeria, fm_oyamakin@yahoo.com

Follow this and additional works at: http://digitalcommons.wayne.edu/jmasm

Part of the Applied Statistics Commons, Social and Behavioral Sciences Commons, and the Statistical Theory Commons

\section{Recommended Citation}

Oyamakin, S. O. (2011) "Height-Diameter Relationship in Tree Modeling Using Simultaneous Equation Techniques in Correlated Normal Deviates," Journal of Modern Applied Statistical Methods: Vol. 10 : Iss. 2 , Article 26.

DOI: $10.22237 /$ jmasm/1320121500

Available at: http://digitalcommons.wayne.edu/jmasm/vol10/iss2/26

This Regular Article is brought to you for free and open access by the Open Access Journals at DigitalCommons@WayneState. It has been accepted for inclusion in Journal of Modern Applied Statistical Methods by an authorized editor of DigitalCommons@WayneState. 


\title{
Height-Diameter Relationship in Tree Modeling Using Simultaneous Equation Techniques in Correlated Normal Deviates
}

\author{
S. O. Oyamakin \\ Forestry Research Institute of Nigeria \\ Ibadan, Nigeria
}

In other to study the complex simultaneous relationships existing in forest/tree growth modeling, six estimation methods of a simultaneous equation model are examined to determine how they cope with varying degrees of correlation between pairs of random deviates using average parameter estimates. A two-equation simultaneous system assumed covariance matrix was considered. The model was structured to have a mutual correlation between pairs of random deviates: a violation of the assumption of mutual independence between pairs of such random deviates. The correlation between the pairs of normal deviates were generated using three scenarios $r=0.0,0.3$ and 0.5 . The performances of estimators considered were examined at various sample sizes $(\mathrm{N}=20,25,30)$ and correlation levels with 50 replications for each. Using the average of parameter estimates criterion, 2 3SLIML were the best estimators followed by FIML and OLS for the three cases studied. Also, as sample size increases from 20 to 25 to 30, 2-3SLIML performed best and was most consistent.

Key words: Growth models, Monte Carlo, random deviates, mutual correlation, average of parameter estimates, simultaneous equation models.

\section{Introduction}

Growth models assist forest researchers and managers in many ways. Some important uses include the ability to predict future yields and to explore silvicultural options. Models provide an efficient way to prepare resource forecasts, but a more important role may be their ability to explore management options and silvicultural alternatives. For example, foresters may wish to know the long-term effect on both the forest and on future harvests, of a particular silvicultural decision, such as changing the cutting limits for harvesting. With a growth model, they can examine the likely outcomes; both with the intended and alternative cutting limits and can make their decision objectively. The process of developing a growth model may also offer

S. O. Oyamakin is a Research Officer in the Department of Planning, Research, Statistics \& Biometrics. Email him at: fm_oyamakin@yahoo.com. interesting new insights into stand dynamics. Forest growth models are very useful to forest managers and forestry researchers in many respects. A forest growth model aims to describe the dynamics of the forest closely and precisely enough to meet the needs of the forester or forestry researcher (dynamics includes all the change processes throughout the forest or tree's lifetime). The primary changes in the forestry field are related to the incorporation, growth and death of trees, a forest's key asset.

There are many forest growth models. Forest models the individual tree. The most common uses of these models for managers are to forecast timber production or, less often, other forestry products (cones, cork, etc.) and to simulate different forestry management alternatives with a view to decision-making. The models help to forecast what long-term effects a forestry management intervention is likely to have on both timber production and the future conditions of the actual forest, as well as the impact of interventions on other forest values. For forestry researchers, models are most useful as tools for researching forest dynamics. 


\section{S. O. OYAMAKIN}

Monte Carlo simulation is a method of analysis based on recreating a chance process (usually with a computer), running it many times, and directly observing the results. The term Monte Carlo method was coined by physicists working on nuclear weapons projects at the Los Alamos National Laboratory. Monte Carlo methods are extensively used in many fields such as operational research, nuclear physics and econometrics, where there are a variety and complexity of problems beyond the available resources of the theoretician (Adepoju, 2009a, c). Many modern investigations have employed Monte Carlo Methods, notable examples include: Wagner (1958); Nagar (1960); Johnston (1972); Anderson \& Sawa (1979); Basmann (1963); Cragg (1966); Anderson (1990); Metropolis (1987); Fomby, Hill \& Johnson (1988); and Smith (1973).

In Monte Carlo studies, data sets are generated with stochastic terms that are free of the problems of multicollinearity, non-spherical disturbances, measurement error and specification error. In the context of a simultaneous equation system, the design of Monte Carlo experiments requires the generation of orthogonal normal deviates or mutually independent sequences distributed as $N(0,1)$. These normal deviates are then transformed to ensure that the disturbance terms are distributed as $N(0, \Sigma)$, which are not serially correlated, where $\Sigma$ is the assumed variance-covariance matrix of the disturbances: However, in real life situations, the errors are not completely correlation free (Adepoju, 2009b; Johnston \& DiNardo, 1984; Anderson \& Sawa, 1973). This study examined the performance of estimators of a two-equation simultaneous model to varying degrees of correlation between pairs of normal deviates.

\section{General Study Framework}

Simultaneous equation models (SEM) are at the heart of a class of models in a data generation process that depends on more than one equation interacting together to produce observed data. Unlike a single-equation model, in which a dependent $(y)$ variable is a function of independent $(x)$ variables, other $y$ variables are among the independent variables in each SEM equation. The $y$ variables in the system are jointly (or simultaneously) determined by the equations in the system.

The following two structural equations are assumed:

$$
Y_{t 1}=\beta_{21} Y_{t 2}+\gamma_{11} X_{t 1}+\gamma_{21} X_{t 2}+U_{t 1}
$$

and

$$
Y_{t 2}=\beta_{12} Y_{t 1}+\gamma_{12} X_{t 1}+\gamma_{32} X_{t 3}+U_{t 2} .
$$

These equations can be rewritten as:

$$
-Y_{t 1}=\beta_{21} Y_{t 2}+\gamma_{11} X_{t 1}+\gamma_{21} X_{t 2}+U_{t 1}
$$

and

$$
\beta_{12} Y_{t 1}=Y_{t 2}+\gamma_{12} X_{t 1}+\gamma_{32} X_{t 3}+U_{t 2} .
$$

These equations are exactly identified.

The reduced form model is derived as

$$
\begin{aligned}
& \beta Y=\Gamma X+U \\
& \Rightarrow Y=\beta^{-1} \Gamma X+\beta^{-1} U \text { i.e } \pi X+V
\end{aligned}
$$

where, $\pi=\beta^{-1} \Gamma$, and by extension, the following endogenous equations are obtained:

$$
\begin{aligned}
Y_{t 1}= & \frac{1}{1-\beta_{21} \beta_{12}}\left(\begin{array}{l}
\gamma_{11} X_{t 1}+\gamma_{21} X_{t 2}+\beta_{21} \gamma_{12} X_{t 1} \\
+\beta_{21} \gamma_{32} X_{t 3}+U_{t 1}+\beta_{21} U_{t 2}
\end{array}\right) \\
Y_{t 2}= & \frac{1}{1-\beta_{21} \beta_{12}}\left(\begin{array}{l}
\gamma_{12} X_{t 1}+\beta_{12} \gamma_{11} X_{t 1}+\beta_{12} \gamma_{21} X_{t 2} \\
+\gamma_{32} X_{t 3}+\beta_{12} U_{t 1}+U_{t 2}
\end{array}\right) \\
Y_{t 1}= & \left(\frac{\gamma_{11}+\beta_{21} \gamma_{12}}{1-\beta_{21} \beta_{12}}\right) X_{t 1}+\left(\frac{\gamma_{21}}{1-\beta_{21} \beta_{12}}\right) X_{t 2} \\
& +\left(\frac{\beta_{21} \gamma_{32}}{1-\beta_{21} \beta_{12}}\right) X_{t 3}+\left(\frac{U_{t 1}+\beta_{21} U_{t 2}}{1-\beta_{21} \beta_{12}}\right)
\end{aligned}
$$

and 


$$
\begin{aligned}
Y_{t 2}= & \left(\frac{\beta_{12} \gamma_{11}+\gamma_{12}}{1-\beta_{21} \beta_{12}}\right) X_{t 1}+\left(\frac{\beta_{12} \gamma_{21}}{1-\beta_{21} \beta_{12}}\right) X_{t 2} \\
& +\left(\frac{\gamma_{32}}{1-\beta_{21} \beta_{12}}\right) X_{t 3}+\left(\frac{\beta_{12} U_{t 1}+U_{t 2}}{1-\beta_{21} \beta_{12}}\right) .
\end{aligned}
$$

Monte Carlo Data Generation

Monte Carlo simulation was used to examine the properties of different statistics computed from sample data. In other words, test-drive estimators were tested, to determine how different recipes perform under different circumstances. The procedure was as follows: In each case an artificial environment was created in which the values of important parameters and the nature of the chance process were specified; then the computer simulated the chance process repeatedly and displayed the results of the experiment.

The main task was the generation of stochastic dependent (endogenous) variables $Y_{i t}(i=1,2 ; t=1, \ldots, T)$, which are subsequently used in estimating the parameters of the model. To achieve this, the following assumptions were necessary:

(i) Values of the predetermined variables $X_{1 t}, X_{2 t}$, and $X_{3 t}(t=1, \ldots, T)$;

(ii) Values of the parameters: $\beta_{12}, \beta_{21}, \gamma_{11}, \gamma_{12}$, $\gamma_{32}$; and

(iii) Values of elements $\Omega$.

The simulation of the error term $U_{i t}(i=1,2, \ldots, T)$ is the most complex step in generating stochastic dependent variables. To conduct the Monte Carlo experiment, first, the sample size $\mathrm{N}$ was specified as $\mathrm{N}=20,25,30$. After specifying the sample size, numerical values were arbitrarily assigned to each structural parameter as follows: $\beta_{12}=1.5$, $\beta_{21}=1.8, \quad \gamma_{11}=1.5, \quad \gamma_{11}=1.5 \quad \gamma_{12}=0.5$, $\gamma_{32}=2.0$ for all cases. The covariance matrix of the disturbances was specified arbitrarily as:

$$
\begin{aligned}
\Omega & =\left(\begin{array}{ll}
\sigma_{11} & \sigma_{12} \\
\sigma_{21} & \sigma_{22}
\end{array}\right) \\
& =\left(\begin{array}{ll}
5.0 & 2.5 \\
2.5 & 3.0
\end{array}\right)
\end{aligned}
$$

The standard random number generator with values obtained from the uniform distribution with mean 0 and standard deviation 1 (Kmenta, 1971) was used to generate the values of the exogenous variables, $X_{i t}(i=1,2,3 ; t=1, \ldots, T)$.

Generation of Random Disturbance Term, U

A 3-stage process was employed to generate random disturbance terms. In the first stage, independent series of normal deviates of required length $(\mathrm{N}=20,25,30)$ were generated. At the second stage, these series were standardized to a normal distribution with mean zero and variance 1. Lastly, the random disturbance terms were generated assuming three degrees of correlation between pairs of random deviates:

(i) Case I: no correlation between the random deviates $\left(r_{\varepsilon_{1}, \varepsilon_{2}}=0\right)$;

Case II: 0.3 correlation level between the random deviates $\left(r_{\varepsilon_{1}, \varepsilon_{2}}=0.3\right)$; and

Case III: 0.5 correlation level between the random deviates $\left(r_{\varepsilon_{1}, \varepsilon_{2}}=0.5\right)$.

The samples sizes considered for each scenario were $\mathrm{N}=20,25$ and 30 . The pairs of random normal deviates based on these sample sizes were generated and each was replicated 50 times. The deviates were then standardized and appropriately transformed to have a specific variance-covariance matrix $\Sigma$ assumed in the model. Numerical values were generated for exogenous variables of the model as described. Next, selected $\left(\varepsilon_{1 t} \varepsilon_{2 t}\right)$ were transformed to be distributed as $N(0, \Sigma)$ where $\Sigma$ was $\operatorname{Cov}\left(U_{t} U_{t}^{\prime}\right)=\Omega \otimes I_{T}$ and elements of $\Omega$ were 


\section{S. O. OYAMAKIN}

decomposed by a non- singular matrix $\rho$ such that $\rho \rho^{\prime}=\Omega$.

$$
\begin{aligned}
& \text { Recall, } V=\beta^{-1} U \\
& \left(\begin{array}{c}
V_{t 1} \\
V_{t 2}
\end{array}\right)=\left(\begin{array}{cc}
\beta^{*} & \beta^{*} \beta_{21} \\
\beta^{*} \beta_{12} & \beta^{*}
\end{array}\right)\left(\begin{array}{c}
U_{t 1} \\
U_{t 2}
\end{array}\right)
\end{aligned}
$$

According to Nagar (1960), $M$ independent terms of standard normal deviates of length $N$ can be transformed into $M$ series of random normal variables with mean 0 and a predetermined covariance matrix. In this model, $M=2$, i.e. $U_{1 t}, U_{2 t}$, if the covariance matrix is

$$
\Omega=\left(\begin{array}{ll}
\sigma_{11} & \sigma_{12} \\
\sigma_{21} & \sigma_{22}
\end{array}\right)
$$

where $\quad \operatorname{var}\left(U_{1}\right)=\sigma_{11}, \quad \operatorname{var}\left(U_{2}\right)=\sigma_{22} \quad$ and $\operatorname{cov}\left(U_{1} U_{2}\right)=\sigma_{12}$, considering both upper and lower triangular matrices. If the upper triangular matrix is

$$
P_{1}=\left(\begin{array}{cc}
\eta_{11} & \eta_{12} \\
0 & \eta_{22}
\end{array}\right)
$$

and the lower triangular matrix is

then

$$
P_{2}=\left(\begin{array}{cc}
\eta_{11} & 0 \\
\eta_{21} & \eta_{22}
\end{array}\right)
$$

$$
\Omega=P_{1} P_{1}^{\prime}=\left(\begin{array}{ll}
\sigma_{11} & \sigma_{12} \\
\sigma_{21} & \sigma_{22}
\end{array}\right)
$$

The pair of standard deviates can be transformed into a pair of random normal variables with mean $\mathrm{Z}^{\mathrm{n}}$ variance $\sigma_{11}, \sigma_{22}$ and covariance $\sigma_{12}$ using

$$
\left[\begin{array}{c}
U_{1 t} \\
U_{2 t}
\end{array}\right]=U_{t}=\eta_{1} \varepsilon_{t}=\left(\begin{array}{cc}
\eta_{11} & \eta_{12} \\
0 & \eta_{22}
\end{array}\right)\left[\begin{array}{l}
\varepsilon_{1 t} \\
\varepsilon_{2 t}
\end{array}\right]
$$

to obtain a pair of random disturbances for the upper triangular matrix:

$$
\begin{aligned}
U_{1 t} & =\eta_{11} \varepsilon_{1 t}+\eta_{12} \varepsilon_{2 t} \\
& =1.707825128 \varepsilon_{1 t}+1.4043
\end{aligned}
$$

and

$$
\begin{aligned}
U_{2 t} & =\eta_{22} \varepsilon_{2 t} \\
& =1.732050808 \varepsilon_{2 t} .
\end{aligned}
$$

where $t=1,2, \ldots, T$. Similarly, an alternative solution can be obtained for the lower triangular matrix:

$$
\begin{aligned}
U_{1 t}^{\prime} & =\eta_{11}^{\prime} \varepsilon_{1 t} \\
& =2.236067978 \varepsilon_{1 t}
\end{aligned}
$$

and

$$
\begin{aligned}
U_{2 t}^{\prime} & =\eta_{12}^{\prime} \varepsilon_{1 t}+\eta_{22}^{\prime} \varepsilon_{2 t} \\
& =1.118033989 \varepsilon_{1 t}+1.322875656 \varepsilon_{2 t} .
\end{aligned}
$$

Generation of Endogenous Variables

Assigning numerical values to the structural parameters provided all values required to generate the endogenous variables. Considering the upper and lower triangular matrix $\mathrm{U}_{\mathrm{t} 1}, \mathrm{U}_{\mathrm{t} 2}$ defined as

$$
\left[\begin{array}{l}
U_{1 t} \\
U_{2 t}
\end{array}\right]=\left(\begin{array}{cc}
1.707825128 & 1.443375673 \\
0 & 1.732050808
\end{array}\right)\left[\begin{array}{l}
\varepsilon_{1 t} \\
\varepsilon_{2 t}
\end{array}\right] \text {, }
$$

and the lower triangular matrix $U^{\prime}{ }_{1 t}, U^{\prime}{ }_{2 t}$ defined as

$$
\left[\begin{array}{c}
U^{\prime}{ }_{1 t} \\
U^{\prime}{ }_{2 t}
\end{array}\right]=\left(\begin{array}{cc}
1.707825128 & 0 \\
1.443375673 & 1.732050808
\end{array}\right)\left[\begin{array}{l}
\varepsilon_{1 t} \\
\varepsilon_{2 t}
\end{array}\right] \text {, }
$$

then, solving $\mathrm{Y}_{\mathrm{t} 1}$ and $\mathrm{Y}_{\mathrm{t} 2}$ using upper triangular matrix results in:

$$
\begin{aligned}
\mathrm{Y}_{1 t}= & -1.411764706 \mathrm{X}_{t 1}-0.588235294 \mathrm{X}_{t 2} \\
& -2.117647059 \mathrm{X}_{t 3}-0.588235294 \mathrm{U}_{t 1} \\
& -0.88235294 \mathrm{U}_{t 2}
\end{aligned}
$$

and 


\section{PERFORMANCE OF SIMULTANEOUS EQUATION MODELING TECHNIQUES}

$$
\begin{aligned}
\mathrm{Y}_{2 t}= & -1.411764706 \mathrm{X}_{t 1}-0.588235294 \mathrm{X}_{t 2} \\
& -2.117647059 \mathrm{X}_{t 3}-0.88235294 \mathrm{U}_{t 1} \\
& -0.588235294 \mathrm{U}_{t 2} .
\end{aligned}
$$

Solving $\mathrm{Y}_{\mathrm{t} 1}$ and $\mathrm{Y}_{\mathrm{t} 2}$ using lower triangular matrix results in:

$$
\begin{aligned}
\mathrm{Y}_{1 t}= & -1.411764706 \mathrm{X}_{t 1}-0.588235294 \mathrm{X}_{t 2} \\
& -2.117647059 \mathrm{X}_{t 3}-0.588235294 \mathrm{U}_{t 1}^{\prime} \\
& -0.88235294 \mathrm{U}_{t 2}^{\prime}
\end{aligned}
$$

and

$$
\begin{aligned}
\mathrm{Y}_{2 t}= & -1.411764706 \mathrm{X}_{t 1}-0.588235294 \mathrm{X}_{t 2} \\
& -2.117647059 \mathrm{X}_{t 3}-0.88235294 \mathrm{U}^{\prime}{ }_{t 1} \\
& -0.588235294 \mathrm{U}_{t 2}^{\prime} .
\end{aligned}
$$

\section{Results}

In theory, and as confirmed by Johnson (1991), when an equation is just identified, estimates of the parameter obtained by 2SLS, 3SLS and LIML should be identical. The results obtained in this study show that 2SLS, 3SLS and LIML estimators yielded virtually identical results, but the OLS, ILS and FIML yielded clearly different results from those estimators. Because 2SLS, 3SLS and LIML have the same results; the estimators shall be denoted as 2-3SLIML.

Analysis of results show that, in case I, 2-3SLIML performed best; it had the closet values to the assumed values in most cases (22) followed by FIML (8 cases) and OLS (5 cases); ILS did not perform at all. Also, as the sample size increased from 20 to 25 to 30 , the value of the estimates moved closer to the true estimates of the parameters in about $72 \%$ of the cases across the upper and lower triangular matrices. For Equation I, the estimates improve from the lower triangular matrices to the upper triangular matrices.

Case II revealed that as the sample size increased, the estimates obtained by 23 SLIML were - in most cases - better than the remaining estimators, which did not show any clear pattern. For both P1 and P2 comparing cases I, II and III across the lower and upper triangular matrices, the performance of estimators under case I was better than those for case II and case III.
Case III revealed that, as the sample size increased from 20 to 25 to 30 , the value of the estimates moved closer to the true estimates of the parameters across the upper and lower triangular matrices. For Equation I, the estimates improve from the lower triangular matrices to the upper triangular matrices.

As an illustration, for OLS over the three magnitudes of the correlation coefficient the estimates of $\beta_{21}$ fell consistently for sample sizes $\mathrm{N}=20,25$ and 30 , that is, column wise comparison for the six estimates:

\begin{tabular}{|l|l|l|l|}
\cline { 2 - 4 } \multicolumn{1}{c|}{} & $\mathrm{N}=20$ & $\mathrm{~N}=25$ & $\mathrm{~N}=30$ \\
\hline Case 1 & 0.92455 & 0.9256 & 0.9286 \\
\hline Case 2 & 0.9105 & 0.9098 & 0.9108 \\
\hline Case 3 & 0.9024 & 0.9045 & 0.9052 \\
\hline
\end{tabular}

A comparison of the three entries in each row shows that estimates rose and fell in CASE 2, and rose consistently in both CASE 1 and CASE 3. Also, along the columns the estimates fell consistently at the three cases of the correlation coefficient at sample sizes $\mathrm{N}=20$, 25 and 30.

The best OLS estimates for $\beta_{21}, \gamma_{11}$ and $\gamma_{21}$ of Equation 1 respectively are: 0.92455 (CASE 1), 0.9256 (CASE 1), 0.9286 (CASE 1) for $\beta_{21}, 0.0077$ (CASE 2), 0.0487 (CASE 2), 0.0323 (CASE 1), for $\gamma_{11 \mathrm{v}}$ and 0.0065 (CASE 2), 0.0594 (CASE 3), 0.0022 (CASE 3) for $\gamma_{21}$. Thus, entries $3(\mathrm{r}=0.0), 0(\mathrm{r}=0.3)$ and $0(\mathrm{r}=$ $0.5)$ under $\beta_{21}, 1(\mathrm{r}=0.0), 2(\mathrm{r}=0.3), 0(\mathrm{r}=0.5)$ under $\gamma_{11}$ and $0(\mathrm{r}=0.0), 1(\mathrm{r}=0.3), 2(\mathrm{r}=0.5)$ under $\gamma_{21}$ (See Table 1).

Similarly, for equation 2, the best OLS estimates for $\gamma_{12}$ are observed for case 1 . Hence, 3( $\mathrm{r}=0.0), 0(\mathrm{r}=0.3)$ and $0(\mathrm{r}=0.5)$. For $\beta_{12}$ they are $0(\mathrm{r}=0.0), 1(\mathrm{r}=0.3 ; 1.0757)$ and 2 $(\mathrm{r}=0.5 ; 1.0944,1.0914)$ and finally, $1(\mathrm{r}=0.0$; $0.06858), 1(\mathrm{r}=0.3 ; 0.0272)$ and $1(\mathrm{r}=0.5$; 0.0955) for $\gamma_{32}$. This is repeated for the other three estimators. Results are displayed in Tables 


\section{S. O. OYAMAKIN}

1 and 2 for P1 and P2 respectively. Hence Tables 1 and 2 reflect the sensitivity of distribution of best estimates to varying correlation coefficients.

Tables 3 and 4 are derived from Tables 1 and 2. Each table contains the correlationbased distribution of estimators which yielded best estimates of not less than 50 percent of the parameters for each equation. Tables 3 and 4 show that CASE 2, where the error term has 0.3 level of correlation, has the least proportion of best estimates and hence fewest so-called best estimators. The most frequent estimator in this interval is the ILS and 2-3SLS.

As shown in Table 5 under $\mathrm{P} 1$, when error terms are not correlated $(\mathrm{r}=0.0)$, OLS, 23SLS and FIML are best for estimating equation 1 , OLS and ILS are good at CASE $2(\mathrm{r}=0.3)$, and 2-3SLS is best at CASE $3(\mathrm{r}=0.5)$. For equation 2, 2-3SLS is best at CASE 1, ILS is best at CASE 2 and FIML performed best at CASE 3. Under P2, the parameters of the first equation are poorly estimated at CASE 2 of the correlation coefficient ( $r=0.3$ ), ILS is best at CASE 1 followed by OLS at CASE 3. Results show that 2-3SLS performed equally well for this equation when the error term is positively correlated as in CASE 3. For equation 2, OLS and ILS are best at CASE 1, 2-3SLS is best at CASE 2 and FIML is best at CASE 3. There is a greater scope of estimating equation 2 at the three cases of correlation coefficient by several estimators.

The scope of estimating the parameter of the first equation is more sensitive to the varying correlation between the error terms than for the equation 2 and this observation is more obvious for P2 than for P1. The ranking of the estimators as displayed in Tables 6 and 8 shows that the estimators rank differently depending on whether the upper (P1) or lower (P2) triangular matrices were used. The ranking also shows that, although ILS ranks highly as the best estimator for the error term with $r=0.0$, OLS is best for the error term with $r=0.3$ and FIML is best for the error term with $r=0.5$. The estimator rankings shown in Table 10, in which $\mathrm{P} 1$ and $\mathrm{P} 2$ are combined, is dominated in part by the ranking obtained under P2. In that table, ILS ranks high in case 1, 2-3SLS in case 2 and FIML ranks high in case 3 where the error terms are positively correlated.

\section{Conclusion}

The finite sampling property of estimators used in this work was the average of parameter estimate. Using the average of parameter estimates criterion, 23 SLIML are the best estimators, followed by FIML and OLS, respectively, for the three cases studied. Also, as the sample size increased from 20 to 25 to $30,2-$ 3SLIML continued to perform best (that is, 2-3 SLIML is consistent); as the sample size increased, the estimates moved closer to the true parameter estimate in most cases. The result of this study will be used to determine the parameter estimation of simultaneous relationships of tree growth models with independent variables like Temperature, rainfall and relative humidity.

\section{References}

Adepoju, A. A. (2009a). Comparative assessment of simultaneous equation techniques to correlated random deviates. European Journal of Scientific Research, 28(2), 253-265.

Adepoju, A. A. (2009b). Performances of the full information estimators in a twoequation structural model with correlated disturbances. Global Journal of Pure and Applied Sciences, 15(1), 101-107.

Adepoju, A. A. (2009c). Comparative performance of the limited information technique in a two-equation structural model. European Journal of Scientific Research, 28(2), 253-265.

Anderson, G. (1980). The structure of simultaneous estimation: A comment. Journal of Econometrics, 14, 271-276.

Anderson, T., \& Sawa, T. (1973). Distributions of estimates of coefficients of a single equation in a simultaneous system and their asymptotic expansions. Econometrica, 41, 683-714.

Anderson, T., \& Sawa, T. (1979). Evaluation of the distribution function of the two- stage least squares estimate. Econometrica, 47, 163-182. 
Table 1: Sensitivity of Estimators Using Average $N=20,25,30, R=50$ (P1)

\begin{tabular}{|c|c|c|c|c|c|c|c|}
\hline \multicolumn{2}{|c|}{} & \multicolumn{3}{|c|}{ Equation 1 } & \multicolumn{3}{c|}{ Equation 2} \\
\cline { 2 - 8 } \multicolumn{2}{|c|}{ Estimators } & $\beta_{21}=1.8$ & $\gamma_{11}=1.5$ & $\gamma_{21}=1.0$ & $\beta_{12}=1.5$ & $\gamma_{12}=0.5$ & $\gamma_{32}=2.0$ \\
\hline \multirow{3}{*}{ OLS } & $\mathrm{C} 1$ & 3 & 1 & 0 & 0 & 3 & 1 \\
& $\mathrm{C} 2$ & 0 & 2 & 1 & 1 & 0 & 1 \\
& $\mathrm{C} 3$ & 0 & 0 & 2 & 2 & 0 & 1 \\
\hline \multirow{3}{*}{ ILS } & $\mathrm{C} 1$ & 1 & 0 & 2 & 1 & 1 & 2 \\
& $\mathrm{C} 2$ & 1 & 2 & 1 & 1 & 2 & 1 \\
& $\mathrm{C} 3$ & 1 & 1 & 0 & 1 & 0 & 0 \\
\hline \multirow{4}{*}{ 2-3SLS } & $\mathrm{C} 1$ & 2 & 2 & 0 & 2 & 1 & 2 \\
& $\mathrm{C} 2$ & 1 & 0 & 0 & 0 & 0 & 1 \\
& $\mathrm{C} 3$ & 0 & 1 & 3 & 1 & 2 & 0 \\
\hline \multirow{3}{*}{ FIML } & $\mathrm{C} 1$ & 1 & 2 & 1 & 1 & 1 & 0 \\
& $\mathrm{C} 2$ & 0 & 1 & 1 & 0 & 1 & 2 \\
& $\mathrm{C} 3$ & 2 & 0 & 1 & 2 & 1 & 1 \\
\hline
\end{tabular}

Table 2: Performance of Estimators Using Average of Parameter Estimate $N=30, R=50(P 2)$

\begin{tabular}{|c|c|c|c|c|c|c|c|}
\hline \multirow{2}{*}{\multicolumn{2}{|c|}{ Estimators }} & \multicolumn{3}{|c|}{ Equation 1} & \multicolumn{3}{|c|}{ Equation 2} \\
\hline & & \multirow{4}{*}{ 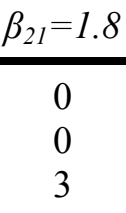 } & \multirow{4}{*}{$\begin{array}{c}\gamma_{11}=1.5 \\
2 \\
1 \\
0\end{array}$} & \multirow{4}{*}{$\frac{\gamma_{21}=1.0}{0} \begin{array}{c}1 \\
2\end{array}$} & \multirow{4}{*}{$\begin{array}{c}\beta_{12}=1.5 \\
3 \\
0 \\
0\end{array}$} & \multirow{4}{*}{ 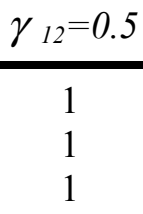 } & \multirow{4}{*}{$\begin{array}{c}\gamma_{32}=2.0 \\
0 \\
2 \\
1\end{array}$} \\
\hline & $\mathrm{C} 1$ & & & & & & \\
\hline OLS & $\mathrm{C} 2$ & & & & & & \\
\hline & C3 & & & & & & \\
\hline & $\mathrm{C} 1$ & 1 & 2 & 2 & 1 & 2 & 1 \\
\hline ILS & $\mathrm{C} 2$ & 0 & 0 & 1 & 0 & 0 & 2 \\
\hline & C3 & 2 & 1 & 0 & 2 & 1 & 0 \\
\hline & $\mathrm{C} 1$ & 0 & 1 & 0 & 1 & 1 & 1 \\
\hline 2-3SLS & $\mathrm{C} 2$ & 1 & 1 & 1 & 2 & 1 & 1 \\
\hline & C3 & 2 & 1 & 2 & 0 & 1 & 1 \\
\hline & $\mathrm{C} 1$ & 1 & 0 & 1 & 1 & 1 & 0 \\
\hline FIML & $\mathrm{C} 2$ & 0 & 1 & 1 & 0 & 1 & 2 \\
\hline & C3 & 2 & 2 & 1 & 2 & 1 & 1 \\
\hline
\end{tabular}




\section{S. O. OYAMAKIN}

Table 3: Correlation-Based Sample Size-Free Distribution of Best Estimators $\mathrm{N}=20,25,30 . \mathrm{R}=50,(\mathrm{P} 1)$

\begin{tabular}{|c|c|c|}
\hline Level of Correlation & Equation 1 & Equation 2 \\
\hline CASE 1 & OLS/2-3SLS/FIML & 2-3SLS/OLS/ILS \\
\hline CASE 2 & - & ILS \\
\hline CASE 3 & 2-3SLS & FIML \\
\hline
\end{tabular}

Source: Table 1

Table 4: Correlation-Based Sample Size-Free Distribution of Best Estimators $\mathrm{N}=20,25,30 . \mathrm{R}=50,(\mathrm{P} 2)$

\begin{tabular}{|c|c|c|}
\hline Level of Correlation & Equation 1 & Equation 2 \\
\hline CASE 1 & ILS & OLS/ILS \\
\hline CASE 2 & - & 2-3SLS \\
\hline CASE 3 & OLS/2-3SLS/FIML & FIML \\
\hline
\end{tabular}

Source: Table 2

Table 5: Sample and Replication-Free Distribution of Best Estimates of P1

\begin{tabular}{|c|c|c|c|c|c|}
\hline \multicolumn{3}{|c|}{ Equation 1 } & \multicolumn{3}{c|}{ Equation 2 } \\
\hline Case 1 & Case 2 & Case3 & Case 1 & Case 2 & Case 3 \\
\hline OLS(4) & OLS(3) & 2-3SLS(4) & 2-3SLS(5) & ILS(4) & FIML(4) \\
\hline 2-3SLS(4) & ILS(3) & FIML(3) & OLS(4) & FIML(3) & OLS(3) \\
\hline FIML(4) & FIML(2) & OLS(2) & ILS(4) & OLS(2) & 2-3SLS(3) \\
\hline ILS(3) & 2-3SLS(1) & ILS(2) & FIML(2) & 2-3SLS(1) & ILS(1) \\
\hline
\end{tabular}

Table 6: Rank of Estimators Using Level of Correlation (P1) for Eq1 and Eq2

\begin{tabular}{|c|c|c|}
\hline Case 1 & Case 2 & Case 3 \\
\hline 2-3 SLS(9) & ILS(7) & 2-3SLS(7) \\
\hline OLS(8) & OLS(5) & FIML(7) \\
\hline ILS(7) & FIML(5) & OLS(5) \\
\hline FIML(6) & 2-3SLS(2) & ILS(3) \\
\hline
\end{tabular}


PERFORMANCE OF SIMULTANEOUS EQUATION MODELING TECHNIQUES

Table 7: Sample and Replication-Free Distribution of Best Estimates of P2

\begin{tabular}{|c|c|c|c|c|c|}
\hline \multicolumn{3}{|c|}{ Equation 1 } & \multicolumn{3}{c|}{ Equation 2 } \\
\hline Case 1 & Case 2 & Case3 & Case 1 & Case 2 & Case 3 \\
\hline ILS(5) & 2-3SLS(3) & OLS(5) & OLS(4) & 2-3SLS(4) & FIML(5) \\
\hline OLS(2) & OLS(2) & 2-3SLS(5) & ILS(4) & OLS(3) & ILS(3) \\
\hline FIML(2) & FIML(2) & FIML(5) & 2-3SLS(3) & FIML(3) & OLS(2) \\
\hline 2-3SLS(1) & ILS(1) & ILS(3) & FIML(2) & ILS(2) & 2-3SLS(2) \\
\hline
\end{tabular}

Table 8: Rank of Estimators Using Level of Correlation (P2) For Eq1 and Eq2

\begin{tabular}{|c|c|c|}
\hline Case 1 & Case 2 & Case 3 \\
\hline ILS(9) & 2-3SLS(7) & FIML(10) \\
\hline OLS(6) & OLS(5) & OLS(7) \\
\hline 2-3SLS(4) & FIML(5) & 2-3SLS(7) \\
\hline FIML(4) & $\operatorname{ILS}(3)$ & $\operatorname{ILS}(6)$ \\
\hline
\end{tabular}

Table 9: Sample and Replication - Free Distribution of Best Estimates of P1 and P2

\begin{tabular}{|c|c|c|c|c|c|}
\hline \multicolumn{3}{|c|}{ Equation 1 } & \multicolumn{3}{c|}{ Equation 2 } \\
\hline Case 1 & Case 2 & Case3 & Case 1 & Case 2 & Case 3 \\
\hline ILS(8) & OLS(5) & 2-3SLS(9) & OLS(8) & ILS(6) & FIML(9) \\
\hline OLS(6) & 1LS(4) & FIML(8) & ILS(8) & FIML(6) & OLS(5) \\
\hline FIML(6) & 2-3SLS(4) & OLS(7) & 2-3SLS(8) & OLS(5) & 2-3SLS(5) \\
\hline 2-3SLS(5) & FIML(4) & ILS(5) & FIML(4) & 2-3SLS(5) & 1LS(4) \\
\hline
\end{tabular}

Table 10: Rank of Estimators Using Level of Correlation (P1 and P2 Combined)

\begin{tabular}{|c|c|c|}
\hline Case 1 & Case 2 & Case 3 \\
\hline ILS(16) & OLS(10) & FIML(17) \\
\hline OLS(14) & ILS(10) & 2-3SLS(14) \\
\hline 2-3SLS(13) & FIML(10) & OLS(12) \\
\hline FIML(10) & 2-3SLS(9) & ILS(9) \\
\hline
\end{tabular}




\section{S. O. OYAMAKIN}

Basmann, R. L. (1963). A note on the exact finite sample frequency functions of generalized classical linear estimators in a leading three-equation case. Journal of the American Statistical Association, 58, 161-171.

Cragg, J. G. (1966). On the sensitivity of simultaneous-equations estimators to the stochastic assumptions of the models. Journal of the American Statistical Association, 61, 136151.

Fomby, T. B., Hill, R. C., \& Johnson, S. R. (1988). Advanced Econometrics methods. New York, NY: Springer-Verlag.

Johnston, J. (1972). Econometric methods, $2^{\text {nd }}$ Edition. New York, NY: McGraw Hill.

Johnston, J., \& DiNardo, J. (1984). Econometric methods, $4^{\text {th }}$ Edition. New York, NY: McGraw-Hill International.

Kmenta, J. (1971). Elements of econometrics. New York, NY: MacMillian.
Nagar, A. (1959). The bias and moment matrix of the general k-class estimators of the parameters in simultaneous equations. Econometrica, 27, 575-595.

Nagar, A. (1960). A Monte Carlo study of alternative simultaneous equation estimators. Econometrica, 28, 573-590.

Metropolis, N. (1987). The beginning of Monte Carlo method. Los Alamos Science Special Issue Dedicated to Stanislaw Ulam, 125130.

Smith, V. (1973). Monte Carlo methods. Lexington, MA: D.C. Health.

Wagner, H. (1958). A Monte Carlo study of estimates of simultaneous linear structural equations. Econometrica, 26, 117-133. 